\title{
Simulation of Magnetic Transition under Influence of Perpendicular Anisotropy Component in Thin Film Longitudinal Recording Media
}

\author{
Toshikazu NISHHHARA, Toshiro ABE, Toshio ANDO, Makoto MIZUKAMI and Masaru SEGAWA \\ Central R\&D Center, JVC \\ 58-7, Shinmei-cho, Yokosuka, Kanagawa 239, Japan
}

\begin{abstract}
Hysteresis properties and transition configurations as related to intergranular exchange coupling and out-of-plane angle of grain's magnetic easy axis have been studied with $3 \mathrm{D}$-static simulation model in longitudinal thin film media. The out-of-plane angle of easy axis contributes to release the bridgings in large exchange coupled thin film media.
\end{abstract}

\section{I . INTRODUCTION}

The studies of magnetic transition noise in thin film recording media have been widely performed with experiments and computer simulations. Particularly the simulation studies based on the full dynamic model (1) (2) have been very successful. However the calculations are extremely computationally intensive. Therefore we have developed static 3D-simulation model for micromagnetic studies.

It is now understood that the transition noise is strongly affected by the degree of exchange coupling between grains ${ }^{(3)}$ (4) in polycrystalline films. Further, present studies have shown that the transition noise is influenced by magnetic longitudinal orientation (B) (B) and oblique orientation ${ }^{(7)}$. Now it is expected that oblique orientation or perpendicular anisotropy component is significant to reduce demagnetization field in thin film longitudinal recording media.

In this paper we describe a newly developed static 3D-simulation method and its application on transition configuration in thin film longitudinal recording media with/without perpendicular anisotropy component and intergranular exchange coupling.

\section{MODEL \& METHOD}

We used basically the Hughes model ${ }^{(8)}$ for grain arrangement and characterization with some modifications. The modeled thin film medium is composed of $25 \times 41$ grains by a two dimentional array. Each grain is a single domain with uniaxial anisotropy and uniform magnetization. The easy axis of each grain is 3D-randomly assigned under specific conditions.

Fig. 1 shows grain's calculation plane. $\mathbf{E}_{11}$ is a grain's easy axis unit vector and $H_{1}$ is a external magnetic field's vector. Magnetic unit vector $M_{1\}}$ is in the plane made from $\mathrm{E}_{\mathrm{i}}$ and $\mathrm{H}_{\mathrm{i},}$. Curling magnetization model
(9) and Stoner-Wohlfarth eq. are used for calculation to magnetic reversal and resulting magnetic direction, respectively.

The external magnetic field $H_{1}$, consists of long range magnetostatic field $\mathrm{Hs}$, nearest neighbor exchange coupling field $\mathrm{He}$ and Karqvist-2D head field Hh.

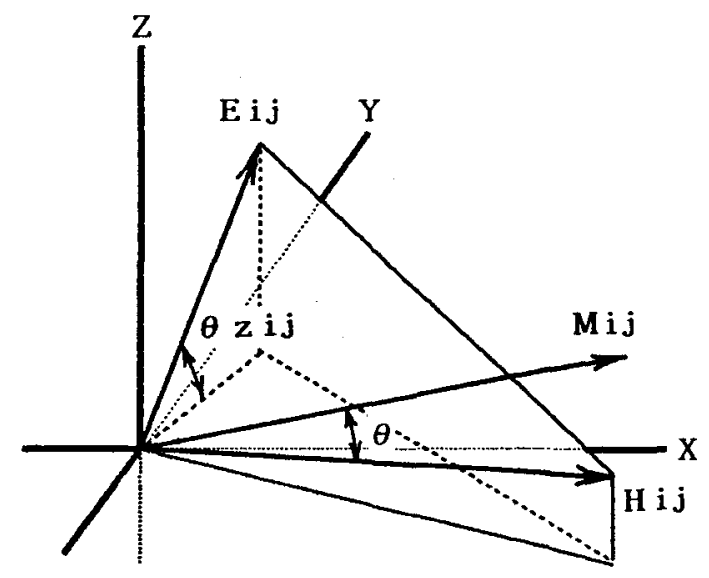

Fig.1 Grain's Calculation Plane

(The direction of plane varies at every calculation step depending on the direction of external magnetic field until convergence is reached. $\mathbf{E}$ is a grain's easy axis unit vector. $\mathbf{H}$ is a external magnetic field vector. $M$ is a magnetic directional unit vector.)

$$
\mathrm{H}_{1\}}=\mathrm{Hs}_{1 j}+\mathrm{He}_{1\}}+\mathrm{Hh}_{1} \text { s }
$$

In case of hysteresis loop calculation, $\mathrm{Hh}$ is de/escalated constantly. The dipole-dipole interaction eq. is used for the long range magnetostatic field $\mathrm{Hs}$

$$
H s_{11}=\Sigma\left(-1 / 4 \pi \mu \text { or }_{1}{ }^{3}\right)\left\{M_{1}-3\left(M_{1}{ }^{*} r_{1}\right) r_{j} / r_{1}{ }^{2}\right\}
$$

The nearest neighbor exchange coupling field $\mathrm{He}_{1 \mathrm{l}}$ is set to be the same equation form as $\left.\mathrm{Hs}_{1}\right\rfloor$

$\mathrm{He}_{\sharp \mathrm{\jmath}}=\mathrm{Ec}^{*} \mathrm{Hs}_{\imath}$,

Here $\mathrm{Ec}_{\mathrm{c}}$ is an exchange coupling constant parameter. In this work Ec is varied from 0 to 0.57 for the study. Ec is selected to be positive or negative value so as to 
cause a magnetic coupling.

The direction of each plane is changed at every calculation step depending on the external magnetic field. The value of unit direction vector $M_{1}$ s is obtained by solving the eqs, of the Stoner-Wohlfarth eq., $\mathrm{dE} / \mathrm{d} \theta=0$ and $\mathrm{d}^{2} \mathrm{E} / \mathrm{d} \theta^{2}>0$. To obtain $M_{1 /}$ self-consistent method $^{(10)}$ is used, and the calculation allows to continue until convergence is reached. The convergence is decided by that all of the value of $M_{1}(x, y, z)$ is within $2 \%$ of prior value of $\mathrm{M}_{1},(\mathrm{x}, \mathrm{y}, \mathrm{z})$.

Numerical conditions are settled as grain size of $20 \mathrm{~nm}$, head gap length $\mathrm{gl}$ of $0.3 \mu \mathrm{m}$ and head medium spacing of $50 \mathrm{~nm}$.

\section{RESULTS AND DISCUSSIONS}

Hysteresis properties and transition configuration results are obtained under the conditions which are varied in ranges of the exchange coupling constant parameter Ec of $0 \sim 0.57$ and the average out-of-plane angle of easy axis $\theta \mathrm{z}= \pm 0, \pm 20, \pm 30, \pm 40$ degrees. This structure is based on actual $\mathrm{Co}-$ alloy/Cr sputtered film media. As for each grain, individual $\mathrm{Ku}$ and out-of-plane angle of easy axis $\theta z_{1}$, are arranged as Gaussian distribution with an average value of $K u$ and $\theta z$, respectively. In $\mathrm{x}-\mathrm{y}$ plane they are set to be oriented randomly. Individual grain's $\mathrm{Ms}, \mathrm{Ku}$, standard deviations of $\mathrm{Ku}$ and $\theta z$, randomness in $\mathrm{x}-\mathrm{y}$ plane and head field are settled to be constant in this work as $\mathrm{Ms}=4000(\mathrm{G}), \mathrm{Ku}=4^{*} 10^{8}$ (erg/cc), standard deviation $\sigma$ of distributed value's $\mathrm{Ku}$ and $\theta z=25 \%$ of each average.

\section{A.Hysteresis Properties}

Fig.2 shows some examples of calculated hysteresis loops for the exchange coupling parameter $E c=0.29$ and for four different out-of-plane angle $\theta \mathrm{z}$. The values of Ms and Hc are normalized. Since the number of grains is limited, lines of hysteresis loops are wobbling.

Fig. 3 and Fig.4 show the calculated results of squareness ratio $\mathrm{Rs}$ and coercivity $\mathrm{Hc}$, respectively, as the functions of out-of-plane angle $\theta_{z}$ and exchange coupling parameter Ec. Rs and Hc values are sensitive to intergranular exchange coupling(1). The larger the exchange coupling, the larger the Rs and Hc in these conditions. The Rs and Hc values are also sensitive to the amount of $\theta \mathrm{z}$. These values decrease in proportion to the grain's out-of-plane angle $\theta \mathrm{z}$, especially in the case of smaller Ec, as shown in Fig.3 and Fig.4. This is caused by the fact that the large $\theta_{z}$ weakens positive interaction among grains.

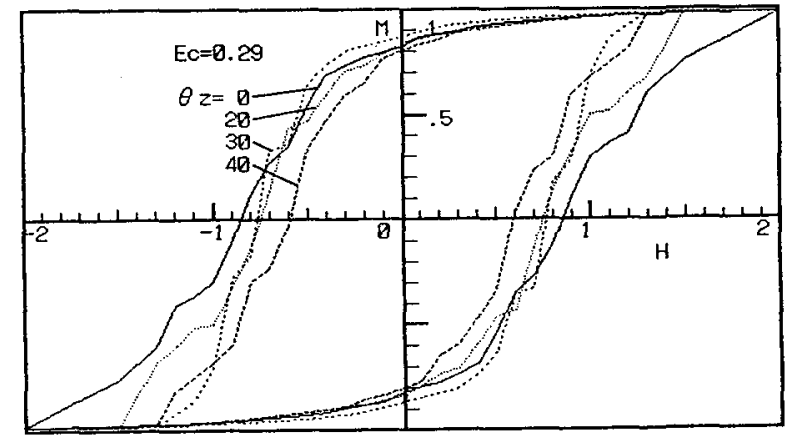

Fig.2 Examples of Calculated Hysteresis Loops (Exchange coupling parameter $\mathrm{Ec}=0.29$, out-of-plane angle $\theta z=0 \sim 40$ degrees)

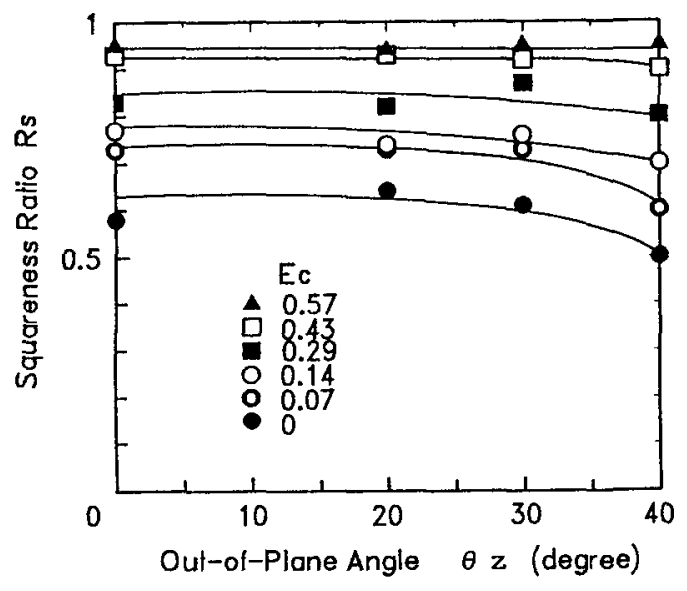

Fig.3 Squareness ratio on out-of-plane angle $\theta z$

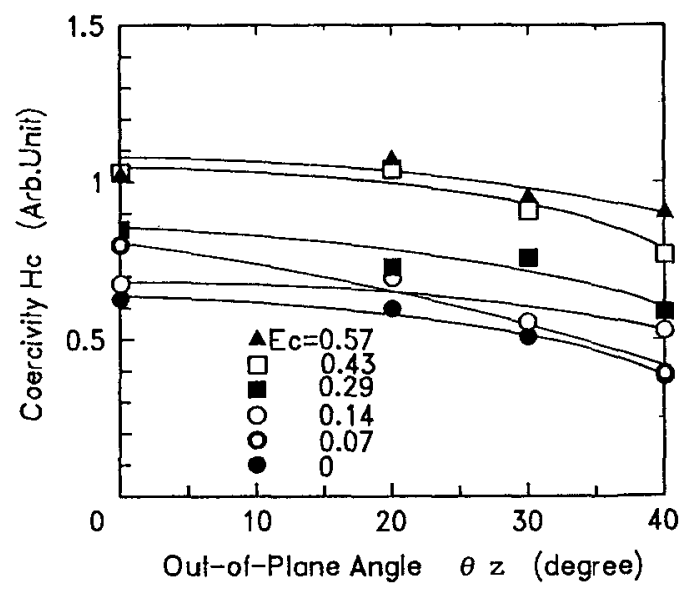

Fig.4 Coercivity on out-of-plane angle $\theta z$ 


\section{B. Transition Configurations}

Transition configurations are studied by simulating the recording of one bit and four-bits transitions. Prior to the recording, the magnetization of each grain is saturated along the recording direction. The head field is set to be about 2.5 times the calculated film coercivity. Periodic boundary conditions are used both in the track direction and in the transverse direction.

Fig.5 and Fig.6 show two typical examples of calculated transition configuration of $\mathrm{x}-\mathrm{y}$ plane for the parameter of $\mathrm{Ec}=0$ and 0.29 , respectively. In these cases out-of-plane angle $\theta z$ is 0 degree. In the value of $\mathrm{Ec}=0$ (Fig.5), obscure pattern can be observed, while in the value of $\mathrm{Ec}=0.29$ (Fig.6) magnetic clusters can be observed. And small bridging also can be observed (pointed with an arrow in Fig.6). Fig.7 shows width of zig-zag domain as the function of exchange coupling parameter Ec from one bit transition configurations. As studied experimentally ${ }^{(11)}$ and numericaly ${ }^{(8)}$, the stronger the Ec, magnetic clusters are formed more distinguished and the wider the width of zig-zag domain.

Fig.8 shows an example of calculated transition configuration of $\mathrm{x}-\mathrm{y}$ plane for the parameter of $\mathrm{Ec}=0.29$ and $\theta \mathrm{z}=20$ degrees. In this case calculated conditions are same as the case of Fig. 6 except the value of $\theta z$. In the value of $\theta z=20$ degrees the bridging pattern from cluster to cluster cannot be observed. And the maximum bit width approaches normal length (marked as $\mathbf{\Delta} \mathbf{\Delta}$ ).

The maximum bit width is defined as a length pointed with " $>$ " in Fig.6 and Fig.8.

Fig.9 shows the width of zig-zag domain calculated by one bit transition as the functions of $\theta \mathrm{z}$ and Ec. Three different patterns are observed in this result. On the first pattern [A], the transition configuration of magnetic media is almost close to that of particulate media that is the case of $\mathrm{Ec}=0 \sim 0.07$. In these cases of $\mathrm{Ec}=0$ and 0.07 the width of zig-zag domain cannot be calculated because of obscure configuration in all values of $\theta \mathrm{z}$.

The larger value of $\theta \mathrm{z}$ causes only the larger randomness of magnetic easy axis distribution in the film plane. The cases of $E c=0.14$, and $\theta z=30,40$ degrees are same as the cases of $E c<0.07$.

Second pattern $[B]$ is the case that Ec has a middle value $(\sim 0.43)$. Grains are formed to magnetic clusters.

But the width of zig-zag domain in the value of $\theta z>20$ degrees is smaller than the case of $\theta z=0$ degree.

Fig.10(a)(b) show cross sectional view of magnetic vector of each grain at the line \#18 in Fig.6 and Fig.8 marked "- - " in each figure, respectively. It can be derived from Fig.10(a)(b) that in the case of $\theta z=0$, magnetic energy can be relaxed only in $x-y$ plane. But in the case of larger $\theta z$, magnetic energy can be relaxed not only in the $x-y$ plane but also in the $x-z$ plane as shown in Fig.10(b) and Fig.8. It is considered that the $\theta z$ of 20 degrees causes the reason of the maximum bit width

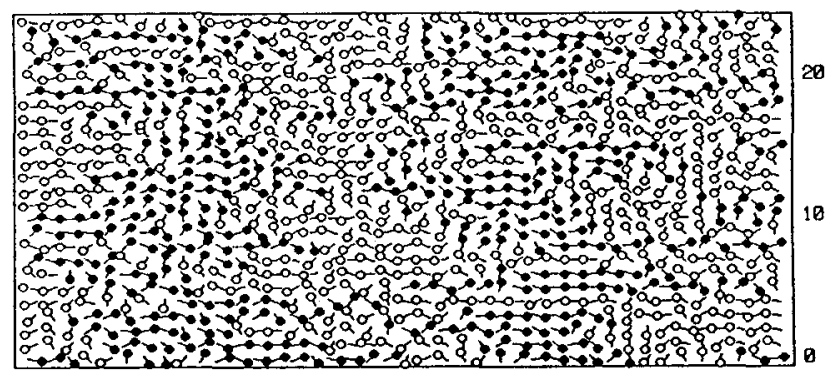

Fig.5 Calculated Transition Configuration (I)

Out-of-plane angle $\theta z=0$ degree. Exchange coupling parameter $E c=0$. An empty circle with a tail represents a magnetization vector with a negative $\mathbf{x}$ component and a solid circle with a tail represents a magnetization vector with a positive $\mathrm{x}$ component.

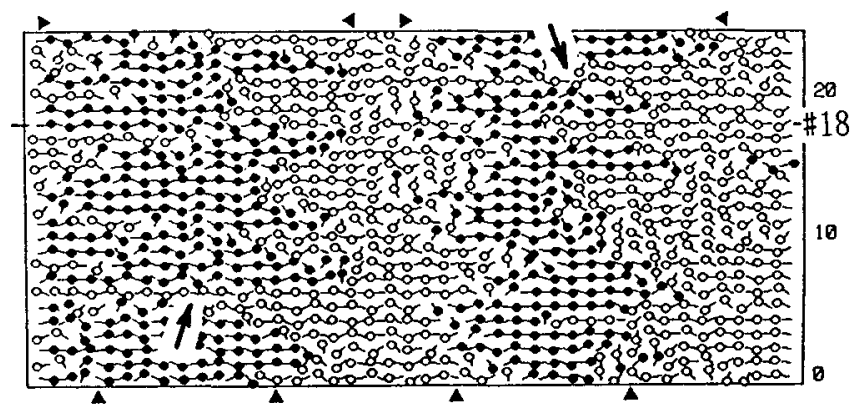

Fig.6 Calculated Transition Configuration (II)

Out-of-plane angle $\theta z=0$ degree. Exchange coupling parameter $E_{\mathrm{c}}=0.29$. An arrow indicates a bridging from cluster to cluster. Figures of right side show line number. Cross sectional view of \#18 is shown in Fig.10(a).

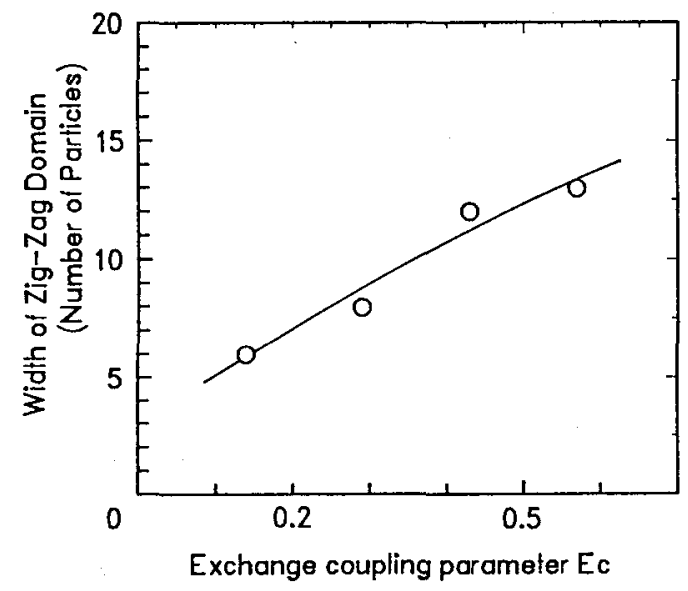

Fig.7 Width of zig - zag domain as a function of Ec ( $\theta z=0$ degree) 
approaching the normal bit length and eliminating the bridging patterns.

In the third case [C] of large Ec $(\sim 0.57)$, large $\theta_{z}$ does not shorten the maximum bit width and eliminate the bridging because of the excessive value of Ec.

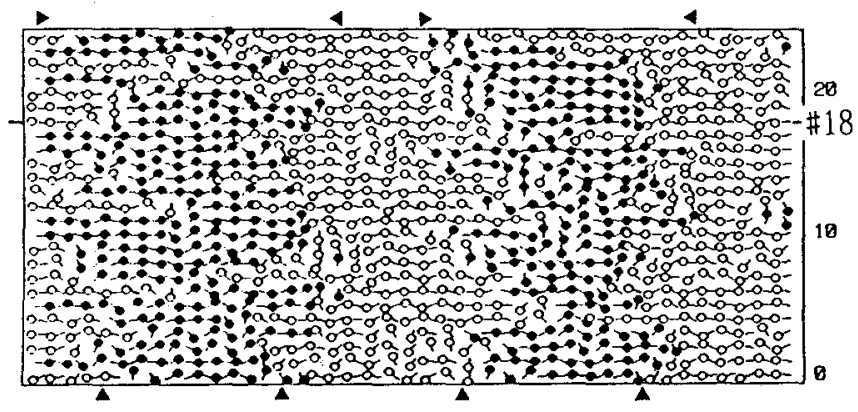

Fig.8 Calculated Transition Configuration (III)

Out-of-plane angle $\theta_{z}=20$ degrees. Exchange coupling parameter $E c=0.29$. Cross sectional view of \#18 is shown in Fig.10(b).

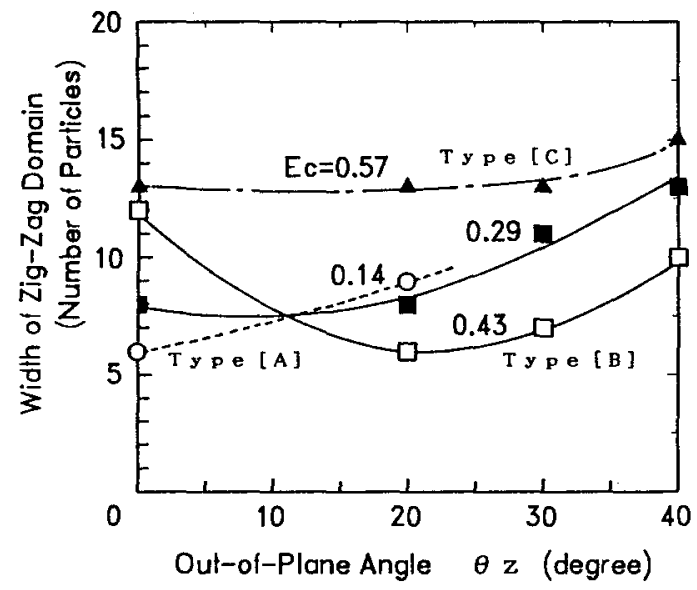

Fig.9 Width of zig-zag domain as a function of $\theta z$

$\ln _{\rightarrow \rightarrow \infty}$

(a)line \#18 in Fig.6

t18

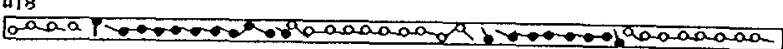

(b)line \#18 in Fig.8

Fig.10 Magnetic $\mathrm{Mx}$ and $\mathrm{Mz}$ component display

(a)line \#18 in Fig.6, (b)line \#18 in Fig.8

\section{CONCLUSION}

The effect of out-of-plane angle of grain's easy axis and intergranular exchange coupling has been studied in thin longitudinal recording film media with the newly developed 3D-static simulation model. In the case of 0 or small value of the exchange coupling, transition configuration is constructed as obscure in planely randomness media. In this case magnetic orientation is important for high density and high $\mathrm{S} / \mathrm{N}$ recording. In the case of larger exchange coupling, grains have formed to magnetic clusters with bridging. In this case out-of-plane angle of grain's easy axis contributes to shorten the zig-zag width and to eliminate the bridging because of relaxation of magnetic energy.

\section{ACKNOWLEDGMENTS}

We would like to thank Drs. Yoshihisa Nakamura, Hiroaki Muraoka, Ikuya Tagawa of Tohoku Univ. for their helpful suggestions and discussions, and Mr.Teruo Takahashi of JVC for his support and encouragement throghout this work.

\section{REFERENCES}

(1)Jian-Gang Zhu and H.Neal Bertram J.Appl.Phys. 63. 3248 (1988)

(2)T.C.Arnoldussen et al Edit. 「Noise in digital Magnetic Recording」(World Scientific) (1992)

(3)Jian-Gang Zhu and H.Neal Bertram IEEE trans. on Magn. vol.24. 6. 2706 (1988)

(4)Tu Chen and Tom Yamashita IEEE tran. on Magn. vol.24. 6. 2700 (1988)

(5)Jian-Gang Zhu IEEE Trans. on Magn. vol.29. 1 195(1993)

(6)Jian-Gang Zhu and Xiao-Guang IEEE Trans. on Magn. vol.29. 1. 324 (1993)

(7)I.Tagawa, Y.Shimizu and Y.Nakamura J.M.S.J. vol.15. S2. 827 (1991)

(8)G.F.Hughes J.Appl.Phys. 545306 (1983)

(9)Y.Nakamura, I.Tagawa and S.Iwasaki J.M.S.J. vol.11. No.2. 119 (1987)

(10)S.Iwasaki and T.Suzuki IEEE trans. on Magn. vol.4. 269 (1968)

(11)S.Miura, T.Yamashita, G.Ching and T.chen IEEE Trans. on Magn. vol.24. 6. 2718 (1988) 